\title{
Adaptation of Cardinal Points Symmetry Landmarks Distribution Model to in-vivo B-Mode Ultrasound Images of Transverse Cross-section of Carotid Arteries
}

\author{
Charles Nnamdi Udekwe and Akinlolu Adediran Ponnle
}

\begin{abstract}
The geometry of the imaged transverse crosssection of carotid arteries in in-vivo B-mode ultrasound images are most times irregular, unsymmetrical, full of speckles and usually non-uniform. We had earlier developed a technique of cardinal point symmetry landmark distribution model (CPSLDM) to completely characterize the Region of Interest (ROI) of the geometric shape of thick-walled simulated B-mode ultrasound images of carotid artery imaged in the transverse plane, but this was based on the symmetric property of the image. In this paper, this developed technique was applied to completely characterize the region of interest of the geometric shape of in-vivo B-mode ultrasound images of non-uniform carotid artery imaged in the transverse plane. In order to adapt the CPS-LD Model to the in-vivo carotid artery images, the single VS-VS vertical symmetry line common to the four ROIs of the symmetric image is replaced with each ROI having its own VS-VS vertical symmetry line. This adjustment enables the invivo carotid artery images possess symmetric properties, hence, ensuring that all mathematical operations of the CPS-LD Model are conveniently applied to them. This adaptability was observed to work well in segmenting the in-vivo carotid artery images. This paper shows the adaptive ability of the developed CPS-LD Model to successfully annotate and segment in-vivo Bmode ultrasound images of carotid arteries in the transverse cross-sectional plane either they are symmetrical or unsymmetrical.
\end{abstract}

Keywords - Cardinal Points, in-vivo B-mode ultrasound, Landmarks, Carotid Artery, Shape Space Pattern.

\section{INTRODUCTION}

Oftentimes, strokes are caused when there is a blockage in an artery leading to the blood supply to the brain being restricted or cut off. Around 20\% of all strokes are caused by carotid artery disease and is more common in older people who have stroke [1].

The carotid arteries are the large arteries which are located at both sides of the neck under the jaw as shown in Fig. 1. Their pulse can be felt as they are quite superficial (about 1 to $3 \mathrm{~cm}$ away from the skin). The carotid artery is divided into three vessels: the common carotid artery (CCA), the external carotid artery (ECA) and the internal carotid artery (ICA). The CCA is the main vessel and bifurcates into two branches: the ICA, which supplies blood to the brain; and the ECA, which supplies blood to the neck and face. This bifurcation is called carotid bulb and it is characterized by an enlargement

Submitted on October 10, 2021.

Published on December 10, 2021.

Charles Nnamdi Udekwe, Federal University of Technology, Akure,

Nigeria. of the vessel [2]. The differentiation of the ICA and ECA is based on their depth from the skin of the neck.

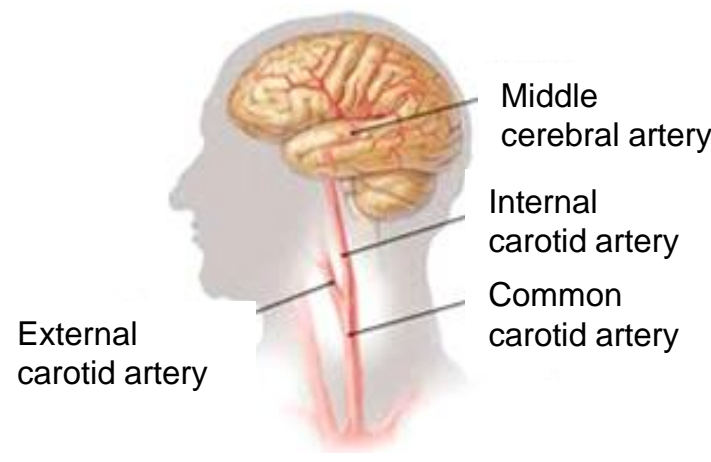

Fig. 1. The position of the Carotid Artery in the neck [3].

In this work, the main vessel which is the CCA is the region where segmentation is carried out. The typical arterial wall of the CCA consists of three layers: an innermost layer, "the intima", a middle layer, "the media", and an outer layer, "the adventita". The intima-media region of the artery is a useful place to examine in the diagnosis of atherosclerosis [4].

In-vivo ultrasound images of carotid arteries especially those in B-mode need the 'eye' of a trained expert to be able to interpret them correctly. The geometry of the carotid arteries in these images is most times irregular, non-uniform, unsymmetrical; and the images are usually full of noise called speckles. The degree of interpretation of these images determines to a large extent the health condition of the patient. The carotid artery imaged in the transverse plane in conjunction with that made in the longitudinal plane help to determine the intima-media thickness (IMT) of the arterial wall. The IMT is a very useful marker for the diagnosis of atherosclerosis especially at its early stage [5]. The transverse image of carotid artery can show to some extent the spread of the plaques in the artery wall, and other applications of it include patient management, genetic research, and therapy evaluation [6]. An in-vivo B-mode ultrasound image of the transverse cross-section of a carotid artery showing the three layers of the arterial wall in the posterior region is shown in Fig. 2.

Several researchers have presented several models and algorithms that can detect and segment the cross-section of the carotid artery in ultrasound images. Some of the works of these researchers are presented as follows: A detection method was developed by [7] for the detection of artery

(e-mail: charlestheory001@gmail.com)

Akinlolu Adediran Ponnle, Federal University of Technology, Akure, Nigeria.

(e-mail: ponnleakinlolu ${ }^{@}$ yahoo.co.uk) 
section area in its perpendicular cut. In their method, the Hough transformation was used for the detection of initial circle parameters while a discrimination-based artificial immune system was used for the classification of pixels located inside and outside of the artery. The main goal of their work was to find the curve of the heart cycle of blood pressure in an ultrasound video-sequence which contains a short sample of pulsating geometric dimensions of the carotid artery in time.

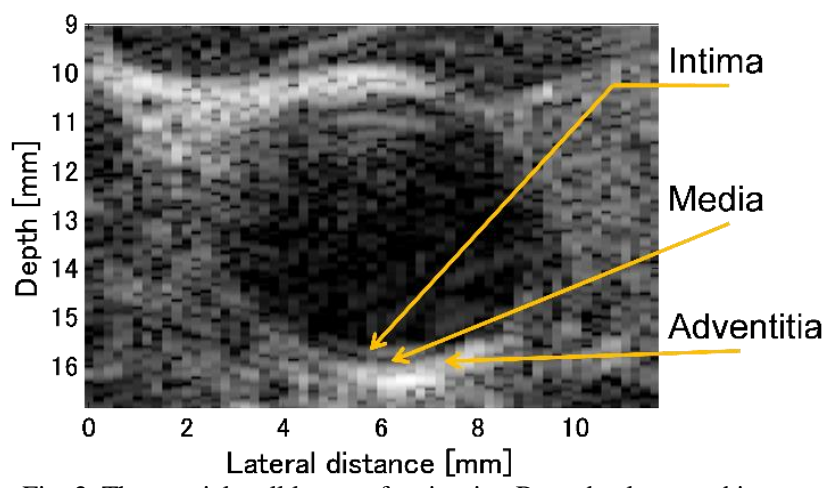

Fig. 2. The arterial wall layers of an in-vivo B-mode ultrasound image of the transverse cross-section of a carotid artery.

A reliable detection of sectional area of an artery during a time in the B-mode ultrasound video sequence frames by automatic monitoring of artery border tissue movement using the Lucas-Canade optical flow technique was developed by [8]. In their method, invariant features that are uniformly selected in the artery border area are tracked in the whole video sequence. Volume cut is then estimated by using fitted ellipse from set of these points so that the most suitable artery shape is denoted in every frame. [9] developed a method for the detection of pulsative circular objects in a medical video sequence. Their motivation for investigating this method was that a circular object is not very apparent in medical images and its detection in such frame is inaccurate. Their proposed method starts from an analysis of movement using optical flow estimation. This was followed by another main processing step; the Hough transform for the circular position estimation. Circles with expected properties are selected using the Bayes classifier and finally, the circle position in a single frame was adapted using the analysis of average pixel intensity in the directions starting from the circle center. A grammar-guided genetic programming (GGGP) automatic localization of common carotid artery (CCA) in ultrasound images was developed by [10]. In their method, the GGGP searches for the best possible combination of simple image processing tasks. The best possible solution is represented with the highest detection precision. The resulting success rate of this method was $82 \%$ which was $4 \%$ higher than the state-of-the-art at that time. [11] developed a method which employed a Viola-Jones detector for efficient detection of transverse sections of the carotid artery. Their algorithm was trained on a set of labelled images using AdaBoost algorithm, Haar-like features, and the Matthews coefficient. Their method had a high success rate, approximately $97 \%$. However, the method was not based on the geometrical description of the object sought.

We had earlier developed the cardinal point symmetry landmark distribution model (CPS-LDM) to completely characterize the ROI of the geometric shape of a transverse cross-section of simulated B-mode ultrasound images of carotid arteries in [12]. We proceeded to apply this developed model on the geometric shape of transverse cross-section of thin-walled phantom carotid arteries in B-mode ultrasound images [13]. In this paper, we model the unsymmetrical invivo arterial wall as a thick-wall and then applied the technique developed in [12], [13] on it to obtain its complete characterization. The model consists of the following components: (1) Cardinal point and inter-cardinal point symmetry description; (2) Fixed landmarks (FLs) equation, movable landmarks (MLs) equation, and the total landmarks (TLs) equation to yield the CPS-LDM equation; and (3) Shape Space Pattern (SSP) of the carotid artery based on the developed CPS-LDM equation.

\section{CARDINAL POINT SYMMETRY (CPS) MODEL FOR IN- VIVO CAROTID ARTERY IMAGE}

\section{A. Data Acquisition}

Real B-mode in-vivo ultrasound images of transverse cross-section of carotid artery of human patients were obtained from the Department of Telecommunication database known as ARTERY_TRANSVERSAL database [7]-[11]. The images have no symmetry at all. Fig. 3 shows a sample of the in-vivo B-mode ultrasound image of transverse cross-section of a carotid artery.

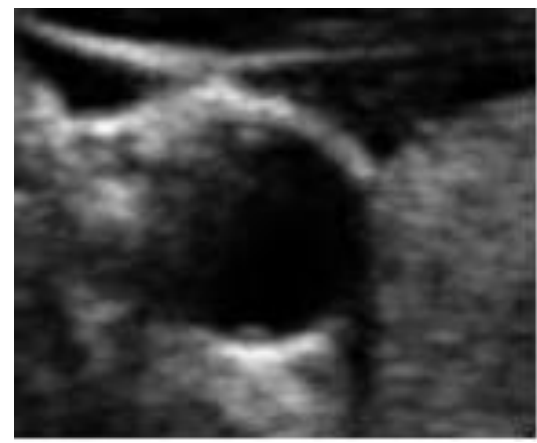

Fig. 3. In-vivo B-mode ultrasound image of a carotid artery in the transverse plane obtained from [11].

\section{B. Arterial Wall Modelling of in-vivo Carotid Arteries}

Due to limitations of in-vivo ultrasound discussed in [13], clearly imaged arterial wall shape in these kind of ultrasound images are not always symmetrical, and this makes it challenging to apply the CPS-LD Model on them. One way this problem was overcome was to 'force' the CPS-LD model developed in [12] on the in-vivo carotid artery images i.e., adapting the in-vivo carotid artery images to possess symmetry such that when the CPS-LD model developed in [12] was used for the segmentation of the ROI on these images, they were found to perform satisfactorily. In order to achieve this, the ROIs in these images were first identified which is the encircled portion shown in Fig. 4 for example. Due to the unsymmetrical nature of the in-vivo carotid artery images, instead of a single VS-VS vertical symmetry line used in [12], each ROI in the in-vivo carotid artery image has its own VS-VS vertical symmetry line drawn as much as possible from the midpoint of each ROI. This is shown in Fig. 5 . 


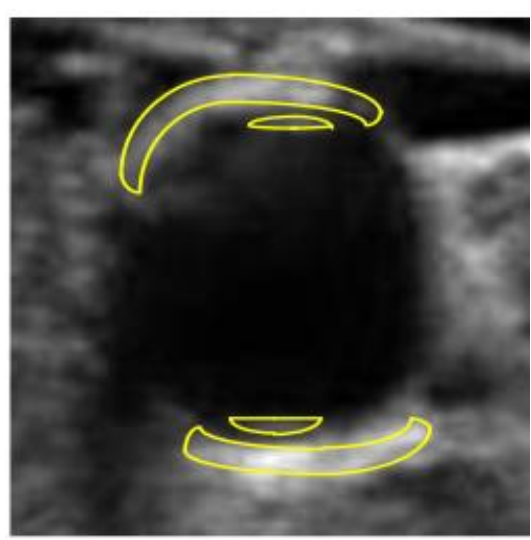

Fig. 4. An in-vivo carotid artery image in the transverse plane showing the ROI.

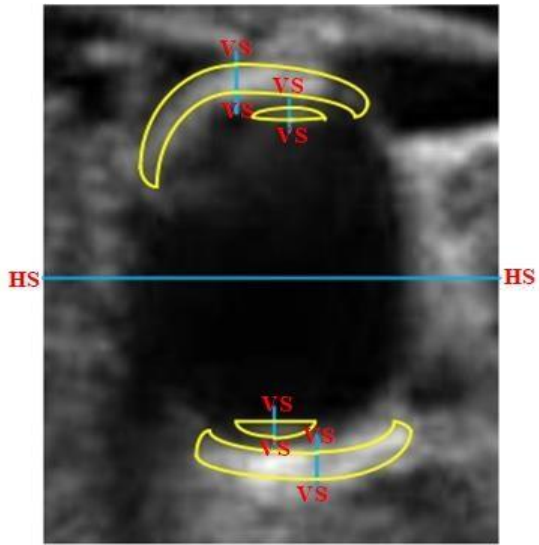

Fig. 5. Each ROI in the in-vivo carotid artery image has its vertical symmetry line.

\section{CPS Model Description}

The cardinal and inter-cardinal points used to develop the CPS-LDM for the in-vivo image are discussed in [13]. The cardinal points concept was then used to describe and label strategic points on the in-vivo image which subsequently led to the full description of the image. This is shown in Fig. 6 and Fig. 7.

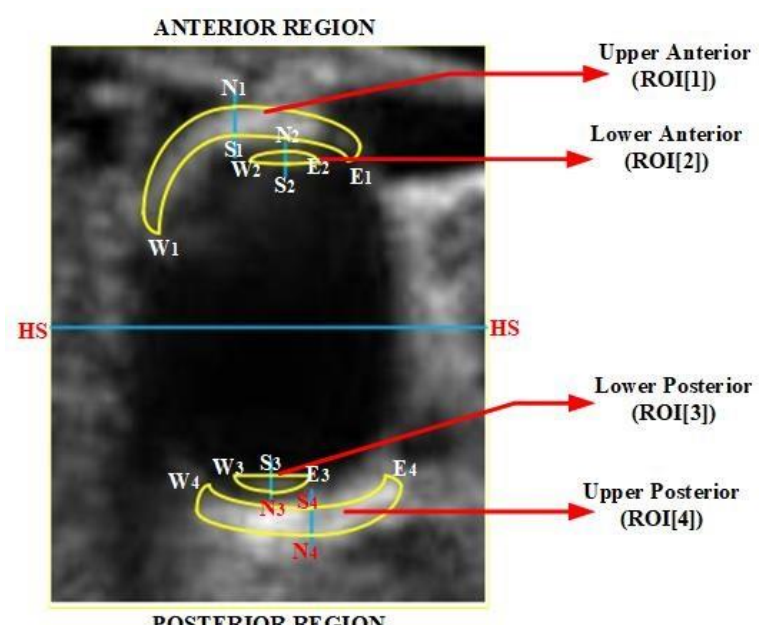

Fig. 6. Fixed cardinal points on the in-vivo carotid artery image.

The description for each point made in [12], [13] for simulated carotid artery and phantom carotid artery images is equally applicable to the points in the image of Figure 6. All other procedures used for segmentation in [12], [13] were adopted to segment the in-vivo carotid artery images.

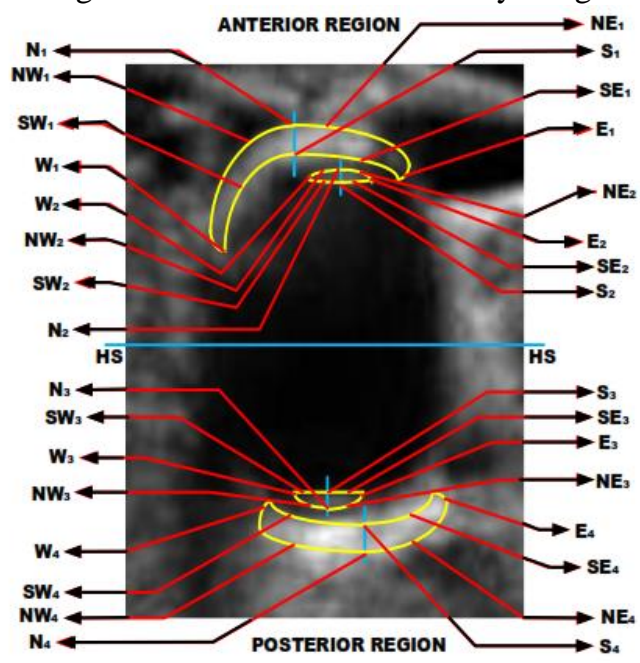

Fig. 7. Full labelling and description of the in-vivo carotid arterial wall image.

\section{ADAPTING THE CPS-LDM MODEL TO THE IN-VIVO CAROTID ARTERY IMAGE}

As formulated in [12], the CPS-LD Model was adapted in this paper to completely characterize the geometry of the invivo carotid artery image despite the fact that it does not have the symmetric properties possessed by the simulated carotid artery image of [12]. Fig. 8 shows how the Fixed Landmarks (FL) were annotated on the in-vivo carotid artery image. Each ROI has its own VS-VS vertical symmetry line unlike the carotid artery in [12] which maintained a single VS-VS vertical symmetry line for all its ROI. The equation that governs the annotation of the FLs on the carotid artery image of Fig. 8 is expressed in (1) [12]:

$$
\mathrm{FLs}=4 \mathrm{U}_{\mathrm{M}}
$$

where $\mathrm{U}$ is the number of ROIs desired to be annotated for segmentation and index M shows the position of the ROIs. The rules followed in selecting the index $\mathrm{M}$ was described in [12]. In order to fully capture the geometry of the ROIs of the in-vivo carotid artery image, there is need to apply the Movable Landmarks (MLs) on it also.

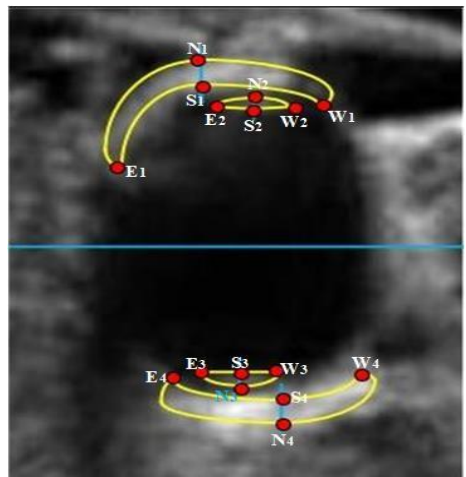

Fig. 8. Positions of the FLs on the carotid artery image.

Using the same notation $n\left(K_{C P}^{R O I}\right)$ as used in [12] to represent the number of integer points that can be used to 
annotate the ROI boundaries of the carotid artery image, the ML equation as developed in [12] is given as (2):

$M L s=n\left(K_{W N E}^{1,4}\right)+n\left(K_{W S E}^{1,4}\right)+n\left(K_{W N E}^{2,3}\right)+n\left(K_{W S E}^{2,3}\right)$

Equation (2) is a series expansion of the notation $n\left(K_{C P}^{R O I}\right)$ where $n(K)$ is the number of integer points on the wall boundaries of the ROIs, the superscript ROI is the position of the ROI under consideration where the ROI positions could be $1,2,3$, or 4 ; and the subscript $\mathrm{CP}$ is the cardinal point region under consideration where $\mathrm{CP}$ could be NW, NE, SW, SE, ..., etc.).

In matrix-like form, equation (2) becomes (3):

$$
M L s=\left[\begin{array}{cc}
K_{W N E}^{1,4} & K_{W N E}^{2,3} \\
K_{W S E}^{1,4} & K_{W S E}^{2,3}
\end{array}\right]
$$

The Total Landmarks (TLs) that can completely annotate the geometry of the in-vivo carotid artery image is governed by equation (4).

$$
\mathrm{TLs}=\mathrm{FLs}+\mathrm{MLs}
$$

Combining equation (1) and (3), equation (4) becomes (5):

$$
\mathrm{TLs}=4 \mathrm{U}_{\mathrm{M}}+\left[\begin{array}{cc}
K_{W N E}^{1,4} & K_{W N E}^{2,3} \\
K_{W S E}^{1,4} & K_{W S E}^{2,3}
\end{array}\right]
$$

IV. APPLICABILITY OF THE CPS-LD MODEL TO IN-VIVO B-MODE CAROTID ARTERY IMAGED IN THE TRANSVERSE PLANE

\section{A. Example 1}

Assuming we want to completely annotate a given in-vivo carotid artery image with 80 landmarks, we want to show how equation (5) can help to accomplish the distribution of landmarks on the in-vivo carotid artery image for annotation which will enable its segmentation.

The Fixed Landmarks FLs are given as equation (1), where $\mathrm{M}=1: 4$ [12]; and ROI 1, 2, 3 and 4 are to be annotated. Hence,

$$
\text { FLs }=4 \times 4=16 \text { landmarks }
$$

Fig. 9 shows the position of the FLs on the in-vivo carotid artery image. ImageJ2 [14] software was used to generate the coordinates of the FLs which was then used to obtain the Shape Space Pattern (SSP) of the image as shown in Fig. 10.

Considering equation (4), subtracting the 16 landmarks used for the FLs from the total 80 landmarks to be used for annotation, we are left with 64 landmarks. The MLs equation of (3) was used to distribute this remaining 64 landmarks.

Since the MLs are at the user's discretion, we assigned the matrix values as follows:

$$
n\left(K_{W N E}^{1,4}\right)=12, n\left(K_{W S E}^{1,4}\right)=12,
$$

$$
n\left(K_{W N E}^{2,3}\right)=4, n\left(K_{W S E}^{2,3}\right)=4
$$

So that $12+12+4+4=64$ MLs as required.

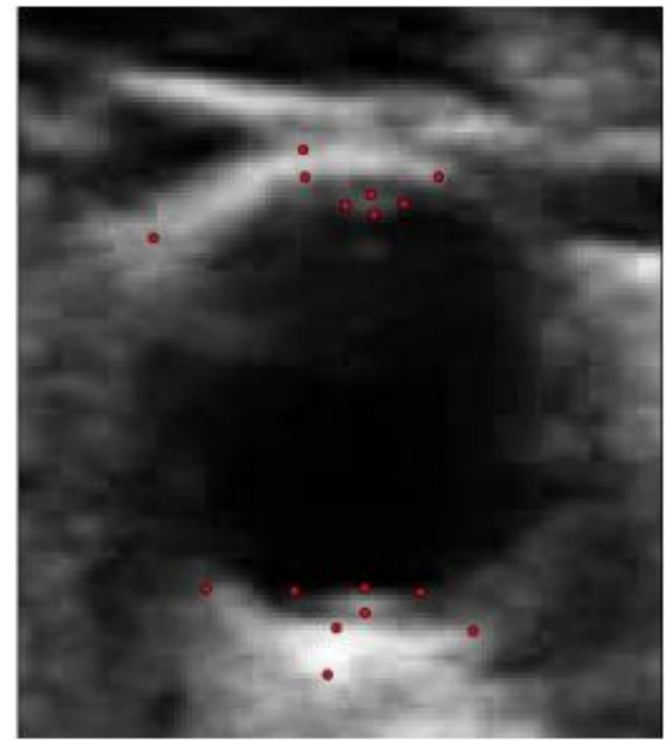

Fig. 9. Positions of the FLs on the in-vivo B-mode ultrasound image of carotid artery in transverse plane based on equation (1)

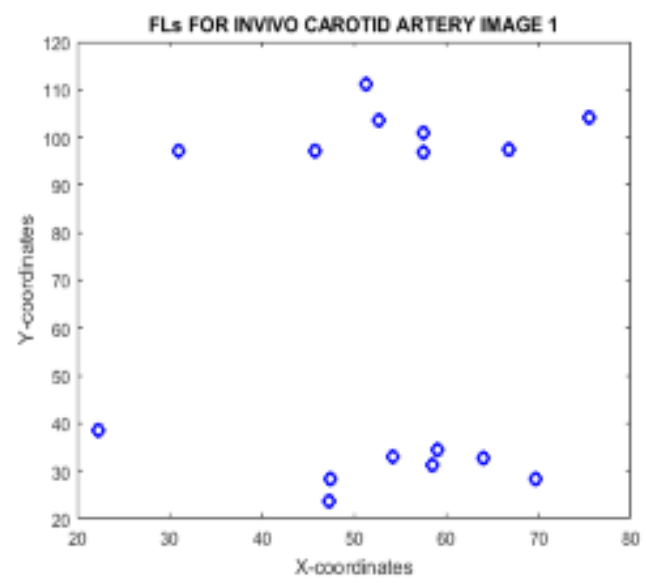

Fig. 10. The SSP for the FLs annotated on the unsymmetrical in-vivo carotid artery image.

The superscript 1, 4 and subscript WNE of the term $n\left(K_{W N E}^{1,4}\right)=12$ implies that ROIs 1 and 4 are divided equally into NW and NE respectively so that along the NW direction. 6 landmarks will be annotated on it and the remaining 6 landmarks will be annotated along the NE direction. This procedure which is applied to the other terms is captured mathematically as given in equations (6) and (7).

$$
\begin{gathered}
M L s=\left[\begin{array}{ll}
R O I 1,4:[W N E]=12 & R O I 2,3:[W N E]=4 \\
R O I 1,4:[W S E]=12 & R O I 2,3:[W S E]=4
\end{array}\right] \\
M L s=\left[\begin{array}{cc}
R O I 1,4: N W=6 & \text { ROI 2,3:NW=2 } \\
R O I 1,4: N E=6 & R O I 2,3: N E=2 \\
R O I 1,4: S W=6 & R O I 2,3: S W=2 \\
R O I 1,4: S E=6 & R O I 2,3: S E=2
\end{array}\right]
\end{gathered}
$$


Fig. 11 shows the distribution of the FLs and MLs on the image using the Image $\mathbf{J} 2$ software.

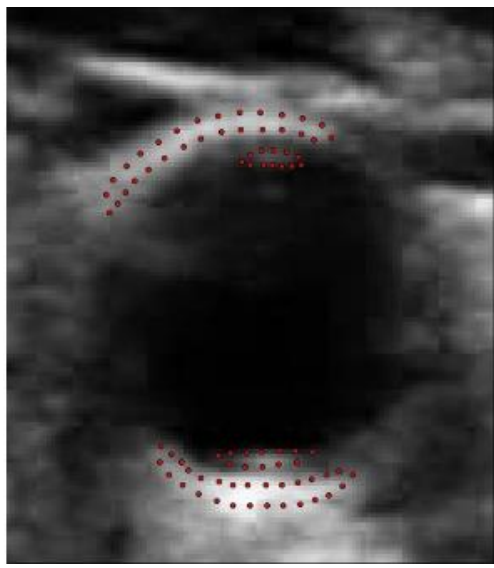

Fig. 11. Landmark distribution for both the FLs and MLs on the in-vivo Carotid Artery Image in transverse plane of Figure 9 (80 landmarks).

The coordinates of the landmarks were also generated from ImageJ2. The generated values are shown in Table I.

\begin{tabular}{|c|c|c|c|c|c|c|c|}
\hline Label & $\begin{array}{c}\text { Land- } \\
\text { mark }\end{array}$ & Xcor & Ycor & Label & $\begin{array}{c}\text { Land- } \\
\text { mark }\end{array}$ & Xcor & Ycor \\
\hline 1 & $\mathrm{FL}$ & 30.0 & 38.0 & 41 & FL & 57.5 & 92.3 \\
\hline 2 & ML & 33.3 & 31.5 & 42 & ML & 61.8 & 94.3 \\
\hline 3 & ML & 39.3 & 27.3 & 43 & ML & 66.8 & 94.5 \\
\hline 4 & ML & 45.5 & 24.0 & 44 & FL & 72.3 & 94.8 \\
\hline 5 & ML & 52.8 & 22.0 & 45 & ML & 78.3 & 94.5 \\
\hline 6 & ML & 66.8 & 20.3 & 46 & ML & 83.3 & 93.8 \\
\hline 7 & ML & 74.3 & 20.0 & 47 & FL & 88.0 & 91.8 \\
\hline 8 & FL & 59.8 & 20.8 & 48 & ML & 83.3 & 91.0 \\
\hline 9 & ML & 80.8 & 20.3 & 49 & ML & 78.3 & 91.3 \\
\hline 10 & ML & 87.3 & 21.0 & 50 & FL & 72.3 & 91.3 \\
\hline 11 & ML & 94.0 & 22.5 & 51 & ML & 67.0 & 91.3 \\
\hline 12 & ML & 100.0 & 24.5 & 52 & ML & 61.5 & 91.5 \\
\hline 13 & ML & 105.8 & 26.8 & 53 & FL & 46.0 & 94.8 \\
\hline 14 & ML & 112.0 & 29.8 & 54 & ML & 47.0 & 98.3 \\
\hline 15 & FL & 114.0 & 35.3 & 55 & ML & 50.5 & 100.8 \\
\hline 16 & ML & 110.5 & 34.5 & 56 & ML & 54.3 & 102.5 \\
\hline 17 & ML & 105.0 & 32.0 & 57 & ML & 58.8 & 103.8 \\
\hline 18 & ML & 99.2 & 29.8 & 58 & ML & 63.3 & 104.0 \\
\hline 19 & ML & 93.3 & 35.3 & 59 & FL & 67.5 & 104.0 \\
\hline 20 & ML & 87.3 & 26.3 & 60 & ML & 72.0 & 104.3 \\
\hline 21 & ML & 80.8 & 25.3 & 61 & ML & 76.8 & 104.3 \\
\hline 22 & FL & 74.5 & 24.8 & 62 & ML & 80.8 & 104.0 \\
\hline 23 & ML & 67.0 & 25.3 & 63 & ML & 84.5 & 103.5 \\
\hline 24 & ML & 60.3 & 26.0 & 64 & ML & 89.0 & 102.3 \\
\hline 25 & ML & 53.8 & 28.0 & 65 & $\mathrm{M}$ & 92.5 & 100.5 \\
\hline 26 & ML & 47.3 & 30.8 & 66 & ML & 95.3 & 98.3 \\
\hline 27 & ML & 41.5 & 33.5 & 67 & FL & 96.5 & 95.3 \\
\hline 28 & ML & 36.5 & 37.5 & 68 & ML & 92.3 & 95.3 \\
\hline 29 & FL & 68.3 & 31.5 & 69 & ML & 89.3 & 96.8 \\
\hline 30 & ML & 70.8 & 30.0 & 70 & ML & 87.3 & 98.3 \\
\hline 31 & ML & 74.8 & 29.3 & 71 & ML & 83.5 & 98.8 \\
\hline 32 & FL & 78.0 & 29.0 & 72 & ML & 80.0 & 99.0 \\
\hline 33 & ML & 82.0 & 29.5 & 73 & ML & 76.5 & 99.0 \\
\hline 34 & ML & 85.5 & 30.3 & 74 & FL & 71.6 & 98.8 \\
\hline 35 & FL & 87.3 & 32.5 & 75 & ML & 67.0 & 98.8 \\
\hline 36 & ML & 85.5 & 32.3 & 76 & ML & 63.5 & 98.8 \\
\hline 37 & ML & 82.0 & 32.3 & 77 & ML & 59.5 & 98.3 \\
\hline 38 & FL & 78.3 & 32.3 & 78 & ML & 55.8 & 97.8 \\
\hline 39 & ML & 74.8 & 32.3 & 79 & ML & 52.0 & 96.0 \\
\hline 40 & ML & 71.0 & 32.3 & 80 & ML & 49.0 & 94.3 \\
\hline
\end{tabular}

From the generated coordinates of the landmarks shown in Table I, the complete Shape Space Pattern (SSP) of the carotid artery image is shown in Fig. 12.

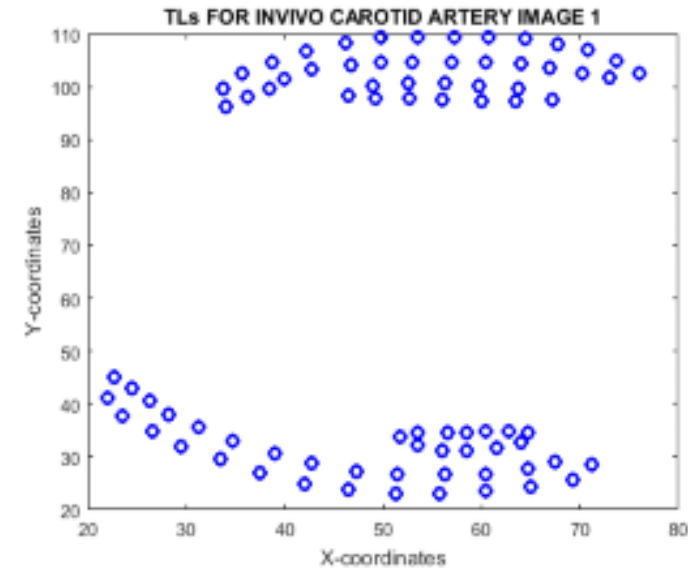

Fig. 12. The complete SSP of the in-vivo Carotid Artery Image of Fig. 10.

\section{B. Example 2}

Given the following equation for another in-vivo B-mode ultrasound image of a carotid artery in the transverse plane;

$$
\mathrm{TLs}=4 \mathrm{U}_{\mathrm{M}}+\left[\begin{array}{ll}
12 & 4 \\
11 & 3
\end{array}\right]
$$

Calculate the TLs required to fully annotate the carotid artery and show its Shape Space Pattern (SSP).

a) Calculations, Results and Discussion

From equation (1), the fixed landmarks are:

$$
\text { FLs }=4 \text { × } 4=16 \text { landmarks }
$$

To obtain the movable landmarks, we have that

$$
\begin{gathered}
n\left(K_{W N E}^{1,4}\right)=12, n\left(K_{W S E}^{1,4}\right)=11, \\
n\left(K_{W N E}^{2,3}\right)=4, n\left(K_{W S E}^{2,3}\right)=3
\end{gathered}
$$

Then

$$
M L s=\left[\begin{array}{cc}
R O I 1,4:[W N E]=12 & R O I 2,3:[W N E]=4 \\
R O I 1,4:[W S E]=11 & R O I 2,3:[W S E]=3
\end{array}\right]
$$

For $n\left(K_{W S E}^{1,4}\right)=11$, symmetry equation demands that SE $=5.5$, and $\mathrm{SW}=5.5$ [12]. However, the number of points must be an integer; hence the symmetry equations are modified to make one side greater than the other side by one integer. For the example above, the following choices can be made

$$
\text { (1) } \mathrm{SE}=5 \text { and } \mathrm{SW}=6
$$

or

(2) $\mathrm{SE}=6$ and $\mathrm{SW}=5$.

Once any of the choice is chosen, it must be consistently maintained for all set of the in-vivo carotid artery images. 
Going for the choice of (11), and making similar procedure and choice for $n\left(K_{W S E}^{1,4}\right)=3$, the MLs equation becomes:

$$
M L s=\left[\begin{array}{cc}
R O I 1,4: N W=6 & R O I 2,3: N W=2 \\
R O I 1,4: N E=6 & R O I 2,3: N E=2 \\
R O I 1,4: S W=6 & R O I 2,3: S W=2 \\
R O I 1,4: S E=5 & R O I 2,3: S E=1
\end{array}\right]
$$

MLs $=(2 \times 6)+(2 \times 6)+(2 \times 2)+(2 \times 2)+(2 \times 6)+(2 \times 5)+(2 \times 2)$ $+(2 \times 1)$

$$
\begin{gathered}
\text { MLs }=12+12+4+4+12+10+4+2 \\
\text { MLs }=60 \text { landmarks. }
\end{gathered}
$$

The total landmarks (TLs) that fully describe the geometry of the in-vivo carotid artery image is calculated thus:

$$
\begin{gathered}
\text { TLs }=\text { FLs }+ \text { MLs } \\
\text { TLs }=16+60 \\
\text { TLs }=76 \text { landmarks }
\end{gathered}
$$

Fig. 13 shows the complete SSP obtained for the in-vivo Bmode ultrasound carotid artery image.

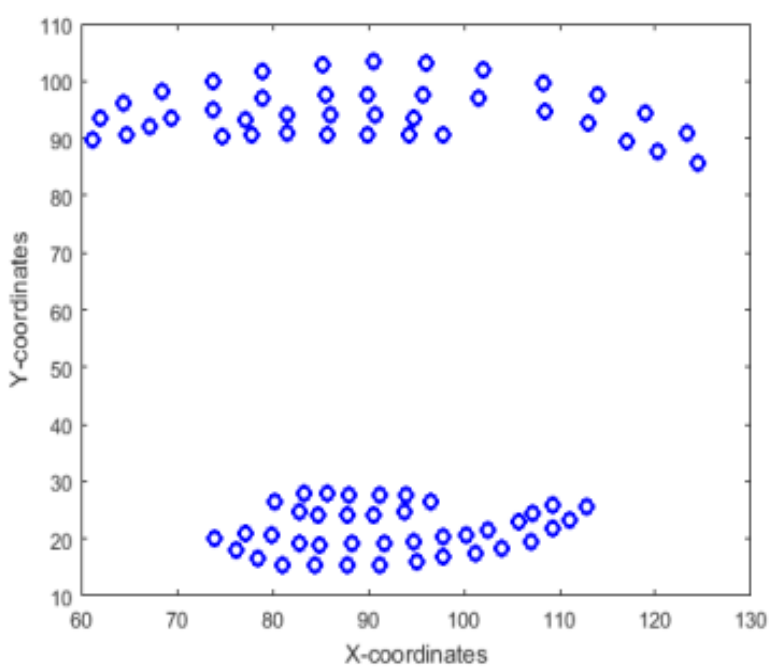

Fig. 13. The complete SSP for the in-vivo B-mode Carotid Artery Image.

\section{CONCLUSION}

In this paper, the Cardinal Point Symmetry Landmark Distribution Model (CPS-LDM) we developed in [12], applied in [13], was adapted to sufficiently segment the ROIs of in-vivo B-mode ultrasound image of thick-walled unsymmetrical carotid artery imaged in the transverse plane by replacing the VS-VS vertical symmetry line to enable it to possess symmetric properties despite that they are unsymmetrical images. This work shows that the CPS-LD model is adaptable to fully annotate both symmetrical and unsymmetrical carotid arteries images in the transverse plane for segmentation.

\section{REFERENCES}

[1] www. stroke.org.uk, "Carotid artery disease," Stroke Association's Information Service, Factsheet 40, version 02, published April 2012.

[2] S. Crisan. Carotid Ultrasound. Medical Ultrasonography, 2011:13:326-330.

[3] https://vascularcures.org/carotid-artery-disease/ "what are the carotid arteries," Vascular Cures, California. Date accessed, $19^{\text {th }}$ of October, 2021.

[4] A. Ponnle, H. Hasegawa, and H. Kanai. Multi element diverging beam from a linear array transducer for transverse cross sectional imaging of carotid artery: simulations and phantom vessel validation. Japanese Journal of Applied Physics (JJAP), 2011;50(7):HF051-HF0510.

[5] T. Mashiyama, H. Hasegawa, and H. Kanai. Designing beam steering for accurate measurement of intima-media thickness at carotid sinus. Japanese Journal of Applied Physics (JJAP), 2006;45(5B):4722-4726.

[6] J. Spence. Technology insight: ultrasound measurement of carotid plaque - patient management, genetic research, and therapy evaluation. Nature Clinical Practice Neurology, 2006;2(11):611-619.

[7] K. Riha, P. Chen, and D. Fu. Detection of artery section area using artificial immune system algorithm. In Proceedings of the 7th WSEAS International Conference on Circuits, Systems, Electronics, Control and Signal Processing, pp. 46-52, 2008.

[8] K. Riha, and I. Potucek. The sequential detection of artery sectional area using optical flow technique. In Proceedings of the 8th WSEAS International Conference on Circuits, Systems, Electronics, Control and Signal Processing, WSEAS Applied Informatics and Communications, pp. 222-226, 2009

[9] K. Riha, and R. Benes. Circle detection in pulsative medical video sequence. In Proceedings of IEEE $10^{\text {th }}$ International Conference on Signal Processing, 2010;1:674-677.

[10] R. Benes, R. Burget, J. Karasek, and K. Riha. Automatically designed machine vision system for the localization of CCA transverse section in ultrasound images. Computer Methods and Programs in Biomedicine, 2013;109(3):92-103.

[11] K. Riha, J. Masek, R. Burget, R. Benes, and E. Zavodna. Novel method for localization of common carotid artery transverse section in ultrasound images using modified Viola Jones detector. Ultrasound in Medicine and Biology, 2013;39(10):1887-1902.

[12] C. N. Udekwe, and A. A. Ponnle. Cardinal points symmetry landmarks distribution model for segmentation of region of interest in simulated B-mode ultrasound carotid artery images. European Journal of Engineering Research and Science (EJERS), 2019;4(5):26-33.

[13] C. N. Udekwe, and A. A. Ponnle. Application of cardinal points symmetry landmarks distribution model to B-mode ultrasound images of transverse cross-section of thin-walled phantom carotid arteries. European Journal of Engineering Research and Science (EJERS), 2019;4(12):96-101.

[14] C. Rueden, J. Schindelin, and M. Hiner. ImageJ2: ImageJ for the next generation of scientific image data. BMC Bioinformatics, 2017;18:529, PMID 29187165.

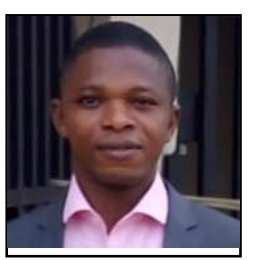

Udekwe C. N. obtained B. Eng. and M. Eng. degrees in Electrical and Electronics Engineering from Federal University of Technology, Akure, Nigeria in 2014, and 2019 respectively.

His research interests include biomedical engineering, digital signal processing, communication systems, electronics, and artificial intelligence. He is currently a postgraduate student in the Department of Electrical and Electronics Engineering of the Federal University of Technology, Akure, Nigeria. He is a student member of Institute of Electrical/Electronics Engineers (IEEE), and a registered engineer with Council of Registered Engineers of Nigeria (COREN). 
Ponnle A. A. obtained B. Eng. and M. Eng. degrees in Electrical and Electronics Engineering from Federal University of Technology, Akure, Nigeria in 1998 and 2003 respectively. He obtained $\mathrm{PhD}$ degree in 2011 from Tohoku University, Japan. His research interests include medical cardiovascular ultrasound imaging, signal processing, communication and electronics engineering, and electromagnetic compatibility. He currently teaches in the Department of Electrical and Electronics Engineering of the Federal University of Technology, Akure, Nigeria where he has been one time the Head of the Department. He is a member of Nigeria Society of Engineers (NSE), and a registered engineer with Council of Registered Engineers of Nigeria (COREN). 